\title{
MODELLING AND FAST NUMERICAL METHODS FOR GRANULAR FLOWS
}

\author{
E. Ferrari, ${ }^{1}$ G. Naldi, ${ }^{2}$ and G. Toscani ${ }^{3}$ \\ ${ }^{1}$ University of Ferrara - Department of Mathematics, Ferrara, Italy, elisa.ferrari@unife.it *, \\ ${ }^{2}$ University of Milan - Department of Mathematics, Milan, Italy, giovanni.naldi@mat.unimi.it \\ ${ }^{3}$ University of Pavia - Department of Mathematics, Pavia, Italy, giuseppe.toscani@unipv.it
}

\begin{abstract}
In this work we discuss the development of fast algorithms for the inelastic Boltzmann equation describing the collisional motion of a granular gas. In such systems the collisions between particles occur in an inelastic way and are characterized by a coefficient of restitution which in the general case depends on the relative velocity of the collision. In the quasi-elastic approximation the granular operator is replaced by the sum of an elastic Boltzmann operator and a nonlinear friction term. Fast numerical methods based on a suitable spectral representation of the approximated model are then presented.
\end{abstract}

keywords: Inelastic Boltzmann equation, Spectral methods, Granular gases, Fast algorithms.

\section{Introduction}

The inelastic Boltzmann equation describes the evolution of materials composed of many small discrete grains, in which the mean free path of the grains is much larger than the typical particle size. Similar as molecular gases, granular gases can in fact be described at a mesoscopic level within the concepts of classical statistical mechanics. Many recent papers (see for example $[1,2,5,12]$ and the references therein), consider Boltzmann-like equations for partially inelastic rigid spheres. Once initialized with a certain velocity distribution, granular gases cool down due to inelastic collisions of their particles. The dissipation of kinetic energy causes a series of non-trivial effects, as formation of clusters and other spatial structures [11], non-Maxwellian velocity distributions, anomalous diffusion, and others.

\footnotetext{
*The financial support of the European network HYKE, funded by the EC as contract HPRN-CT-200200282 and of the project NUMSTAT 2005, Comitato dei Sostenitori, funded by the University of Ferrara is acknowledged.
}

Please use the following format when citing this chapter:

Ferrari, E., Naldi, G., and Toscani, G., 2006, in IFIP International Federation for Information Processing, Volume 202, Systems, Control, Modeling and Optimization, eds. Ceragioli, F., Dontchev, A., Furuta, H., Marti, K., Pandolfi, L., (Boston: Springer), pp. 151-161. 
In a granular gas, the microscopic dynamics of grains is governed by the restitution coefficient $h$ which relates the normal components of the particle velocities before and after a collision. If one assumes that the grains are identical perfect spheres of diameter $\sigma>0,(x, v)$ and $(x-\sigma n, w)$ are their states before a collision, where $n \in \mathbf{S}^{2}$ is the unit vector along the center of both spheres, the post collisional velocities $\left(v^{*}, w^{*}\right)$ are such that

$$
\left(v^{*}-w^{*}\right) \cdot n=-h((v-w) \cdot n) .
$$

Thanks to (1), and assuming the conservation of momentum, one finds the change of velocity for the colliding particles as

$$
v^{*}=v-\frac{1}{2}(1+h)((v-w) \cdot n) n, \quad w^{*}=w+\frac{1}{2}(1+h)((v-w) \cdot n) n .
$$

For elastic collisions one has $h=1$, while for inelastic collisions $h$ decreases with increasing degree of inelasticity. In the first part of this note we briefly review the basic ideas behind the kinetic modelling of dissipative collisions.

From a numerical viewpoint, similarly to the classical rarefied gas dynamics case, the solution of the inelastic Boltzmann equation represents a real challenge. This is mostly due to the high dimensionality of the equation but also to the inelastic collision dynamics which preclude the use of the fast Boltzmann solvers recently presented in [2] for elastic collisions. We will see in the last part of this note, how in the quasi-elastic approximation we can recover fast algorithms also in the inelastic case.

\section{Modelling dissipative collisions}

The main difference between the classical Boltzmann equation for elastic rigid spheres and its dissipative version is contained in the binary collision among particles. In (2) the only parameter which can contain the description of the inelastic collision is the coefficient of restitution.

In the literature, essentially for simplicity, the restitution coefficient is frequently assumed to be a physical constant. A constant restitution coefficient however does not describe realistic situations. In fact, the restitution coefficient may depend on the relative velocity in such a way that collisions with small relative velocity are close to be elastic. The simplest physically correct description of dissipative collisions is based on the assumption that the spheres are composed by viscoelastic material, which is in good agreement with experimental data. The velocity-dependent restitution coefficient for viscoelastic spheres of diameter $\sigma>0$ and mass $m$ reads

$$
h=1-C_{1} A \alpha^{2 / 5}|(v-w) \cdot n|^{1 / 5}+C_{2} A^{2} \alpha^{4 / 5}|(v-w) \cdot n|^{2 / 5} \pm \ldots
$$


with

$$
\alpha=\frac{3 \sqrt{3}}{2} \frac{\sqrt{\sigma} Y}{m\left(1-\nu^{2}\right)},
$$

where $Y$ is the Young modulus, $\nu$ is the Poisson ratio, and $A$ depends on dissipative parameters of the material. The constant $C_{1}$ and $C_{2}$ can be explicitly computed. The impact velocity dependence (3) of the restitution coefficient $h=h((v-w) \cdot n)$ has been recently obtained by generalizing Hertz's contact problem to viscoelastic spheres. We skip here details that can be found in the literature (see [5] and the references therein). What is important in what follows, is that real situations of microscopic collisions between grains can be described in general assuming that the coefficient of restitution satisfies

$$
1-h=2 \bar{\beta} \gamma(|(v-w) \cdot n|),
$$

where $\gamma(\cdot)$ is a given function and $\bar{\beta}$ is a parameter which is small in presence of small inelasticity. For example, for small values of $\alpha$, the velocity dependence of the restitution coefficient in a collision of viscoelastic spheres can be expressed at the leading order as in (5), choosing $\gamma(r)=r^{1 / 5}$.

\section{The Boltzmann equation}

Following the standard procedures of kinetic theory [8], the evolution of the distribution function can be described by the Boltzmann-Enskog equation for inelastic hard spheres, which for the force-free case reads [4]

$$
\frac{\partial f}{\partial t}+v \cdot \nabla_{x} f=G(\rho) \bar{Q}(f, f)(x, v, t),
$$

where $\bar{Q}$ is the so-called granular collision operator, which describes the change in the density function due to creation and annihilation of particles in binary collisions

$$
\bar{Q}(f, f)(v)=4 \sigma^{2} \int_{\mathbf{R}^{3}} \int_{\mathbf{S}_{+}^{2}}(q \cdot n)\left\{\chi f\left(v^{* *}\right) f\left(w^{* *}\right)-f(v) f(w)\right\} d w d n
$$

In (6)

$$
\rho(x, t)=\int_{\mathbf{R}^{3}} f(x, v, t) d v
$$

is the density, and the function $G(\rho)$ is the statistical correlation function between particles, which accounts for the increasing collision frequency due to the excluded volume effects. We refer to [7] for a detailed discussion of the meaning of the function $G$.

In (7), $q=(v-w)$, and $\mathbf{S}_{+}^{2}$ is the hemisphere corresponding to $q \cdot n>0$. The velocities $\left(v^{* *}, w^{* *}\right)$ are the pre-collisional velocities of the so-called inverse collision, which results with $(v, w)$ as post-collisional velocities. The factor $\chi$ 
in the gain term appears respectively from the Jacobian of the transformation $d v^{* *} d w^{* *}$ into $d v d w$ and from the lengths of the collisional cylinders $h\left|q^{* *} \cdot n\right|=$ $|q \cdot n|$. For a constant restitution coefficient, $\chi=h^{-2}$. This enlightens a second remarkable difference between the elastic and the inelastic collision operators. While the Jacobian of the elastic collision is equal to unity, allowing for the exchangeability of the rule of the pre- and post-collisional velocities, in the inelastic case the Jacobian is different from unity, and this implies a different role of pre- and post-collisional quantities.

To avoid the presence of the function $\chi$, and to study approximations to the granular operator (7) it is extremely convenient to write the operator (7) in weak form. More precisely, let us define with $<\cdot, \cdot>$ the inner product in $L_{1}\left(\mathbf{R}^{3}\right)$. For all smooth functions $\varphi(v)$, it holds

$$
\begin{aligned}
& <\varphi, \bar{Q}(f, f)>=4 \sigma^{2} \int_{\mathbf{R}^{3}} \varphi(v) \bar{Q}(f, f)(v) d v= \\
& 2 \sigma^{2} \int_{\mathbf{R}^{3}} \int_{\mathbf{R}^{3}} \int_{\mathbf{S}^{2}}|q \cdot n|\left(\varphi\left(v^{*}\right)-\varphi(v)\right) f(v) f(w) d v d w d n .
\end{aligned}
$$

The last equality follows since the integral over the hemisphere $\mathbf{S}_{+}^{2}$ can be extended to the entire sphere $S^{2}$, provided the factor $1 / 2$ is inserted in front of the integral itself. In fact changing $n$ into $-n$ does not change the integrand.

Let $\left(v^{\prime}, w^{\prime}\right)$ be the post collisional velocities in a elastic collision with $(v, w)$ as incoming velocities,

$$
v^{\prime}=v-(q \cdot n) n, \quad w^{\prime}=w+(q \cdot n) n .
$$

Following [18], we rewrite the inelastic collision (2) in terms of the elastic collision (9) obtaining

$$
v^{*}=v^{\prime}+\frac{1}{2}(1-h)(q \cdot n) n, \quad w^{*}=w^{\prime}-\frac{1}{2}(1-h)(q \cdot n) n .
$$

If we assume that the coefficient of restitution can be described at the leading order by (5),

$$
v^{*}-v^{\prime}=\bar{\beta} \gamma(|q \cdot n|)(q \cdot n) n
$$

Let us consider a Taylor expansion of $\varphi\left(v^{*}\right)$ around $\varphi\left(v^{\prime}\right)$. Thanks to (11) we get

$$
\varphi\left(v^{*}\right)=\varphi\left(v^{\prime}\right)+\ddot{\beta} \nabla \varphi\left(v^{\prime}\right) \cdot \gamma(|q \cdot n|)(q \cdot n) n+O\left(\bar{\beta}^{2}\right)
$$

If the collisions are nearly elastic, $\bar{\beta}<<1$, and we can cut the expansion (12) after the first-order term. Inserting (12) into (20) gives

$$
<\varphi, \bar{Q}(f, f)>=<\varphi, Q(f, f)>+\bar{\beta}<\varphi, I(f, f)>.
$$


It is a simple matter to recognize that in (13) $Q(f, f)$ is the classical Boltzmann collision operator for elastic hard spheres molecules [8],

$$
Q(f, f)(v)=2 \sigma^{2} \int_{\mathbf{R}^{3}} \int_{\mathbf{S}^{2}}|q \cdot n|\left\{f\left(v^{\prime}\right) f\left(w^{\prime}\right)-f(v) f(w)\right\} d w d n
$$

In fact, the velocity $v^{\prime}$ into (13) is obtained from $(v, w)$ through the elastic collision (9).

Let us now study in more detail the second contribution to the inner product (13). Using the properties of the transformation (9), we obtain

$$
\begin{aligned}
& <\varphi, I(f, f)>= \\
& 2 \sigma^{2} \int_{\mathbf{R}^{3}} d v \varphi(v) \operatorname{div}_{v} \int_{\mathbf{R}^{3}} \int_{\mathbf{S}^{2}} n(q \cdot n)|q \cdot n| \gamma(|q \cdot n|) f\left(v^{\prime}\right) f\left(w^{\prime}\right) d w d n .
\end{aligned}
$$

In fact, the transformation $d v d w$ into $d v^{\prime} d w^{\prime}$ given by (9) is such that $q^{\prime} \cdot n=$ $-q \cdot n$, while its Jacobian is equal to unity. The last equality follows from the divergence theorem. This shows that the granular correction is the nonlinear friction operator $\bar{\beta} I(f, f)(v)$, where

$$
I(f, f)(v)=2 \sigma^{2} \operatorname{div}_{v} \int_{\mathbf{R}^{3}} \int_{\mathbf{S}^{2}} n(q \cdot n)|q \cdot n| \gamma(|q \cdot n|) f\left(v^{\prime}\right) f\left(w^{\prime}\right) d w d n .
$$

Finally, for nearly elastic granular collisions, with a restitution coefficient satisfying (5), the Enskog-Boltzmann equation can be modelled at the leading order as

$$
\frac{\partial f}{\partial t}+v \cdot \nabla_{x} f=G(\rho) Q(f, f)(x, v, t)+G(\rho) \bar{\beta} I(f, f)(x, v, t),
$$

where $Q$ is the classical elastic Boltzmann collision operator, and $I$ is a dissipative nonlinear friction operator which is based on elastic collisions between particles.

\section{Fast methods}

In this section we restrict ourselves to the study of the space homogeneous case

$$
\frac{\partial f}{\partial t}=G(\rho) Q(f, f)(v, t)+G(\rho) \bar{\beta} I(f, f)(v, t)
$$

This is motivated by the use of a splitting argument in the numerical solution of the kinetic equation. It is clear that all the main numerical difficulties are contained in the right hand side of (18). Here we will use a Carleman-like representation of the operators $Q(f, f)$ and $I(f, f)$, together with a suitable angular approximation, in order to derive spectral methods that can be evaluated 
through fast algorithms. We refer to $[2,10,15]$ and references therein for further details on fast spectral methods.

For the sake of simplicity we will first derive the method for the classical operator $Q(f, f)$ and then we briefly describe how to extend it to the nonlinear friction term $I(f, f)$.

\subsection{A Carlemann-like representation}

Let us use the identity

$$
\int_{\mathrm{S}^{2}}(u \cdot n)_{+} \varphi(n(u \cdot n)) d n=\frac{|u|}{4} \int_{\mathrm{S}^{2}} \varphi\left(\frac{u-|u| n}{2}\right) d n,
$$

in order to write collision operator $Q(f, f)$ in the form

$$
Q(f, f)(v)=\frac{\sigma^{2}}{2} \int_{\mathbf{R}^{3}} \int_{\mathbf{S}^{2}}|q|\left\{f\left(v^{\prime}\right) f\left(w^{\prime}\right)-f(v) f(w)\right\} d w d n
$$

where now

$$
v^{\prime}=\frac{1}{2}(v+w)+\frac{1}{2}|q| n, \quad w^{\prime}=\frac{1}{2}(v+w)-\frac{1}{2}|q| n .
$$

Then we use a Carlemann-like representation which conserves more symmetries of the collision operator when one truncates it in a bounded domain.

As explained in [2] the basic identity we shall need is

$$
\frac{1}{2} \int_{\mathbf{S}^{2}} F(|u| n-u) d n=\frac{1}{|u|} \int_{\mathbf{R}^{3}} \delta\left(2 x \cdot u+|x|^{2}\right) F(x) d x .
$$

Using (22) with $u=q=v-w$ and performing the change of variables $x \rightarrow x / 2$ and $w \rightarrow y=w-v-x$ we can write

$$
\begin{aligned}
Q(f, f)(v)= & 2 \sigma^{2} \int_{x \in \mathbf{R}^{3}} \int_{y \in \mathbf{R}^{3}} \delta(x \cdot y) \\
& {[f(v+y) f(v+x)-f(v+x+y) f(v)] d x d y . }
\end{aligned}
$$

Now let us consider the bounded domain $\mathcal{D}_{T}=[-T, T]^{3}(0<T<+\infty)$. Next we have to truncate the integration in $x$ and $y$ without affecting the action of the operator for compactly supported functions. Thus we set them to vary in $\mathcal{B}_{S}$, the ball of center 0 and radius $S$. For a compactly supported function $f$ with support $\mathcal{B}_{R}$, we take $S=2 R$ in order to obtain all possible collisions. In fact we have

$$
|x|^{2} \leq|x|^{2}+|y|^{2}=|x+y|^{2}=|q|^{2} \leq(2 R)^{2},
$$

thus $|x| \leq 2 R$ and similarly we get $|y| \leq 2 R$. 
The operator now reads

$$
\begin{aligned}
Q^{R}(f, f)(v)= & 2 \sigma^{2} \int_{x \in \mathcal{B}_{2 R}} \int_{y \in \mathcal{B}_{2 R}} \delta(x \cdot y) \\
& {[f(v+y) f(v+x)-f(v+x+y) f(v)] d x d y, }
\end{aligned}
$$

with $v \in \mathcal{B}_{\sqrt{2} R}$. The interest of this representation is to preserve the real collision kernel and its invariance properties. The next step consist in a suitable periodization of the operator on $\mathcal{D}_{T}$ which prevents intersections of the regions where $f$ is different from zero. Note that in (23) the arguments of the integrands are contained into $\mathcal{B}_{3 \sqrt{2}} R$. In fact we have that $|x| \leq 2 R$ and $|y| \leq 2 R$ imply $|x+y|^{2}=|x|^{2}+|y|^{2} \leq 8 R^{2}$ (thanks to the orthogonality condition $x \cdot y=0$ consequence of the $\delta$ function) and then $|x+y| \leq 2 \sqrt{2} R$. From this we get $|v+x+y| \leq|v|+|x+y| \leq \sqrt{2} R+2 \sqrt{2} R=3 \sqrt{2} R$. Thus we need to take $T \geq(3+\sqrt{2}) R / \sqrt{2}$ as a bound for the periodization.

\subsection{Spectral methods and fast algorithms}

Now we use the representation $Q^{R}$ to derive the spectral methods. In the rest of the paragraph, for simplicity, we take $G(\rho)=1$ and we neglect the friction correction setting $I(f, f)=0$ into (18). Following the same computation as in the classical spectral method [15] but using representation (23) we obtain the following set of ordinary differential equations on the Fourier coefficients

$$
\frac{d \hat{f}_{k}(t)}{d t}=\sum_{\substack{l, m=-N \\ l+m=k}}^{N} \hat{\beta}(l, m) \hat{f}_{l} \hat{f}_{m}, \quad k=-N, \ldots, N
$$

where now $\hat{\beta}(l, m)=\beta(l, m)-\beta(m, m)$ with

$$
\beta(l, m)=2 \sigma^{2} \int_{x \in \mathcal{B}_{2 R}} \int_{y \in \mathcal{B}_{2 R}} \delta(x \cdot y) e^{i l \cdot x} e^{i m \cdot y} d x d y .
$$

In the sequel we shall focus on $\beta$, and one easily checks that $\beta(l, m)$ depends only on $|l|,|m|$ and $|l \cdot m|$.

The search for fast deterministic algorithms for a collision operator in $\mathbf{R}^{d}$, i.e. algorithms with a cost lower than $O\left(N^{2 d+\kappa}\right)$ (with typically $\kappa=1$ ), consists mainly in identifying some convolution structure in the operator. If this is trivial for the loss part of the operator, for the gain part this is rather contradictory with the search for a conservative scheme in a bounded domain, since the boundary condition needed to prevent for the outgoing or ingoing collisions breaks the invariance. 
The aim is to approximate each $\hat{\beta}(l, m)$ by a sum

$$
\beta(l, m) \simeq \sum_{p=1}^{A} \alpha_{p}(l) \alpha_{p}^{\prime}(m) .
$$

This gives a sum of $A$ discrete convolutions and so the algorithm can be computed in $O\left(A N^{d} \log _{2} N\right)$ operations by means of standard FFT techniques. To this purpose we shall use a further approximated collision operator where the number of possible directions of collision is reduced to a finite set.

We start from representation (23) and write $x$ and $y$ in spherical coordinates

$$
\begin{aligned}
& Q^{R}(f, f)(v)=\frac{\sigma^{2}}{2} \int_{e \in \mathbf{S}^{2}} \int_{e^{\prime} \in \mathrm{S}^{2}} \delta\left(e \cdot e^{\prime}\right) d e d e^{\prime} \\
& \left\{\int_{-R}^{R} \int_{-R}^{R} \rho \rho^{\prime}\left[f\left(v+\rho^{\prime} e^{\prime}\right) f(v+\rho e)-f\left(v+\rho e+\rho^{\prime} e^{\prime}\right) f(v)\right] d \rho d \rho^{\prime}\right\} .
\end{aligned}
$$

Let us denote with $\mathcal{A}$ a discrete set of orthogonal couples of unit vectors $\left(e, e^{\prime}\right)$, which is even, i.e. $\left(e, e^{\prime}\right) \in \mathcal{A}$ implies that $\left(-e, e^{\prime}\right),\left(e,-e^{\prime}\right)$ and $\left(-e,-e^{\prime}\right)$ belong to $\mathcal{A}$ (this property on the set $\mathcal{A}$ is required to preserve the conservation properties of the operator). Now we define $Q^{R, \mathcal{A}}$ to be

$$
\begin{aligned}
& Q^{R, \mathcal{A}}(f, f)(v)=\frac{\sigma^{2}}{2} \int_{\left(e, e^{\prime}\right) \in \mathcal{A}}\left\{\int_{-R}^{R} \int_{-R}^{R} \rho \rho^{\prime}\right. \\
& \left.\left[f\left(v+\rho^{\prime} e^{\prime}\right) f(v+\rho e)-f\left(v+\rho e+\rho^{\prime} e^{\prime}\right) f(v)\right] d \rho d \rho^{\prime}\right\} d \mathcal{A}
\end{aligned}
$$

where $d \mathcal{A}$ denotes a discrete measure on $\mathcal{A}$ which is also even in the sense that $d \mathcal{A}\left(e, e^{\prime}\right)=d \mathcal{A}\left(-e, e^{\prime}\right)=d \mathcal{A}\left(e,-e^{\prime}\right)=d \mathcal{A}\left(-e,-e^{\prime}\right)$. It is easy to check that $Q^{R, \mathcal{A}}$ has the same conservation properties as $Q^{R}$.

By taking a spherical parametrization $(\theta, \varphi)$ of $e \in \mathbf{S}_{+}^{2}$ and uniform grids of respective size $M_{1}$ and $M_{2}$ for $\theta$ and $\varphi$, we get

$$
\beta(l, m) \simeq \frac{2 \sigma^{2} \pi^{2}}{M_{1} M_{2}} \sum_{p, q=0}^{M_{1}, M_{2}} \alpha_{p, q}(l) \alpha_{p, q}^{\prime}(m),
$$

where

$$
\begin{aligned}
& \alpha_{p, q}(l)=\phi_{R}^{3}\left(l \cdot e_{\left(\theta_{p}, \varphi_{q}\right)}\right), \quad \alpha_{p, q}^{\prime}(m)=\psi_{R}^{3}\left(\Pi_{e_{\left(\theta_{p}, \varphi_{q}\right)}}(m)\right) \\
& \phi_{R}^{3}(s)=\int_{-R}^{R} \rho e^{i \rho s} d \rho, \quad \psi_{R}^{3}(s)=\int_{0}^{\pi} \sin \theta \phi_{R}^{3}(s \cos \theta) d \theta
\end{aligned}
$$


and

$$
\left(\theta_{p}, \varphi_{q}\right)=\left(\frac{p \pi}{M_{1}}, \frac{q \pi}{M_{2}}\right) .
$$

Typically we shall consider this expansion with $M=M_{1}=M_{2}$ to avoid anisotropy in the computational grid. The computational cost of the algorithm is then $O\left(M^{2} N^{3} \log _{2} N\right)$, compared to $O\left(N^{6}\right)$ of the usual spectral method. Thus one requires $M^{2} \log N \ll N^{3}$ in order to speed up the schemes.

\subsection{Extension to the nonlinear friction}

Finally, by applying an analogous procedure, it's possible to approximate the friction operator $I(f, f)$ in (17) by its truncated version $I^{R}(f, f)$ and to extend the above spectral method to the full problem (18). The computations are similar and hereafter are shortly summarized. The idea is that one just applies the same representation and truncation as for $Q$, inside the divergence of $I$. The result has exactly the same form with another kernel. More precisely using identity (19) we can write

$$
I(f, f)(v)=\frac{\sigma^{2}}{2} \operatorname{div}_{v} \int_{\mathbf{R}^{3}} \int_{\mathbf{S}^{2}}\left(\frac{|q|-|q| n}{2}\right)|q| \gamma(|q|) f\left(v^{\prime}\right) f\left(w^{\prime}\right) d w d n
$$

Next the Carlemann-like representation is obtained through (22) and yields

$$
I(f, f)(v)=2 \sigma^{2} \operatorname{div}_{v} \int_{\mathbf{R}^{3}} \int_{\mathbf{R}^{3}} x \gamma(|x|) \delta(x \cdot y) f(v+x) f(v+y) d x d y .
$$

Periodization on $\mathcal{D}_{T}$ then gives

$$
I^{R}(f, f)(v)=2 \sigma^{2} \int_{\mathcal{B}_{2 R}} \int_{\mathcal{B}_{2 R}} \delta(x \cdot y) \gamma(|x|) x \cdot \nabla_{v}(f(v+x) f(v+y)) d x d y
$$

where now the arguments of the integrand are supported into $\mathcal{B}_{(2+\sqrt{2}) R}$.

The major difference is that the resulting kernel is characterized by the vector

$$
x \gamma(|x|)
$$

This kernel clearly decouples since it does not depend on $y$ and so the resulting spectral scheme, similarly to the previous section, can be computed with fast algorithms.

Thus, for the full model (18) we obtain the Fourier coefficients

$$
\frac{d \hat{f}_{k}(t)}{d t}=G(\rho) \sum_{\substack{l, m=-N \\ l+m=k}}^{N}\left(\hat{\beta}(l, m)+\bar{\beta} \beta_{I}(l, m)\right) \hat{f}_{l} \hat{f}_{m}, \quad k=-N, \ldots, N
$$


where $\hat{\beta}(l, m)$ are given by (25) and the nonlinear friction coefficients are

$$
\beta_{I}(l, m)=2 \sigma^{2} i k \cdot \int_{x \in \mathcal{B}_{2 R}} \int_{y \in \mathcal{B}_{2 R}} x \gamma(|x|) \delta(x \cdot y) e^{i l \cdot x} e^{i m \cdot y} d x d y .
$$

We omit the details of the fast solver which follows the lines of the one described for the elastic Boltzmann equation [9, 10, 2].

\section{Conclusions}

In this note we have summarized some recent results related to the modelling of granular gases and the development of fast algorithms. In particular we have seen how the method recently developed in [2] can be extended to nonlinear friction equations and to the quasi-elastic approximation even for non constant coefficient of restitution.

\section{References}

[1] N. Bellomo, M. Esteban, M. Lachowicz. Nonlinear kinetic equations with dissipative collisions. Appl. Math. Letters 8:46-52, 1995.

[2] D. Benedetto, E. Caglioti, M. Pulvirenti. A kinetic equation for granular media. $M 2 A N$ Math. Model. Numer. Anal. 31:615-641, 1997.

[3] D. Benedetto, E. Caglioti, J.A. Carrillo, M. Pulvirenti. A non-maxwellian steady distribution for one-dimensional granular media. J. Statist. Phys. 91:979-990, 1998.

[4] A.V. Bobylev, J.A. Carrillo, I.M. Gamba. On some properties of kinetic and hydrodynamic equations for inelastic interactions J. Statist. Phys. 98:743-773, 2000.

[5] N.V. Brilliantov, T. Pöschel. Granular gases with impact-velocity dependent restitution coefficient. In Granular Gases: 100-124, Lecture Notes in Physics, Vol. 564, SpringerVerlag, Berlin, 2000.

[6] J.A. Carrillo, C. Cercignani, I.M. Gamba. Steady states of a Boltzmann equation for driven granular media. Phys. Rev. E (3) 62:7700-7707, 2000.

[7] C. Cercignani. Recent developments in the mechanism of granular materials. Fisica Matematica e ingegneria delle strutture, Pitagora Editrice, Bologna, 1995.

[8] C. Cercignani, R. Illner, M. Pulvirenti. The mathematical theory of dilute gases. Applied Mathematical Sciences, Vol. 106, Springer-Verlag, New-York, 1994.

[9] F.Filbet, C. Mouhot, L. Pareschi. Solving the Boltzmann equation in $N \log _{2} N$. SISC (to appear), 2005.

[10] F.Filbet, C. Mouhot, L. Pareschi. Work in progress.

[11] I. Goldhirsch. Scales and kinetics of granular flows. Chaos 9:659-672, 1999.

[12] S. McNamara, W.R. Young. Kinetics of a one-dimensional granular medium in the quasielastic limit. Phys. Fluids A 5:34-45, 1993.

[13] C. Mouhot, L. Pareschi. Fast algorithms for computing the Boltzmann collision operator. Math. Comp. (to appear), 2005.

[14] G. Naldi, L. Pareschi, G. Toscani. Spectral methods for one-dimensional kinetic models of granular flows and numerical quasi elastic limit. M2AN Math. Model. Numer. Anal. $37: 73-90,2003$ 
[15] L. Pareschi, G. Russo. Numerical solution of the Boltzmann equation. Spectrally accurate approximation of the collision operator. SIAM J. Numer. Anal. 37:1217-1245, 2000.

[16] L. Pareschi, G. Toscani. Modelling and numerics of granular gases. In Modeling and computational methods for kinetic equations: 259-285, Series Model. Simul. Sci. Eng. Technol., Birkhauser, Boston, 2004.

[17] G. Toscani. One-dimensional kinetic models of granular flows, M2AN Math. Model. Numer. Anal. 34:1277-1292, 2000.

[18] G. Toscani. Kinetic and hydrodinamic models of nearly elastic granular flows, Monatsch. Math. 142:179-192, 2004. 